\title{
Towards the Deployment of a Centralized ICT Architecture in the Automotive Domain
}

\author{
Hauke Stähle, Ljubo Mercep, Alois Knoll \\ Lehrstuhl für Robotik und Eingebettete Systeme \\ Technische Universität München \\ Garching bei München, Germany
}

\author{
Gernot Spiegelberg \\ Institute for Advanced Study \\ Technische Universität München/Siemens AG \\ Garching bei München, Germany
}

\begin{abstract}
The effort for the integration of new functionalities in today's vehicles is increasing as the interconnection and verification of the growing amount of heterogeneous and distributed electric control units (ECU's) becomes more difficult. The demand for a new architectural approach that can cope with the increasing complexity and offers possibilities for a smooth integration of future technologies is urgent. Such technologies are drive-by-wire systems or advanced driver assistance systems. This paper extends the previously introduced ICT architecture for future vehicles by the analysis of a possible system, hardware and software architecture and their properties. In addition, a migration path from the current vehicle architecture is suggested and economic impacts of suggested improvements are shown. A short description of differences to AUTOSAR is given. Demonstrators for proof-of-concept and evaluation are also discussed. With this work we have brought the previously described ICT architecture one step closer to the large-scale implementation in the automotive domain.
\end{abstract}

Keywords: Automotive Systems, Software and Hardware Architectures, Embedded Systems

\section{INTRODUCTION}

Information and communication technology (ICT), in the form of electrics, electronics, and software in vehicles, is already essential for the competitiveness in the automotive industry. Its most notable effects are the improvement of driving performance and comfort, and the enhancement of both passive and active safety [1]. As most of the functions in today's cars are realized with software components, the demand for processing power and data bandwidth increases dramatically. At the same time, the integration effort increases, as the impact of the existing functions is harder to model and predict. Only extensive verification can overcome this issue, leading to cost explosion. We present an extended approach, that is suited for the upcoming needs of increased functionality in the automotive area.

An overview of the architecture in today's cars and the involved problems is given in section II. The building blocks of the centralized ICT architecture approach are introduced in section III while possible migration paths are discussed in section IV. Section V gives an overview of economic impacts and section VI introduces the demonstrators used for proof-ofconcept and evaluation. The paper is concluded and an outlook is given in section VII.

\section{ICT ARCHITECTURE IN TODAY'S VEHICLES}

The hardware and software architecture in today's vehicles was developed in an evolutionary way. The existing systems were modified, extended and interconnected with new components to be able to fulfill new functionalities in a car. ICT and especially software were the main drivers for new innovations. The lines of code increased from 100 in the $1970 \mathrm{~s}$ up to as much as ten million lines today [2]. It is expected that this trend will continue and most innovations in a car are contributed by software components. The evolutionary development process led to several problems:

- Heterogeneous networks: Various networks are incorporated in one car. This makes the development and integration process more challenging, as different paradigms have to be combined, such as priority-based communication via $\mathrm{CAN}$ and time-triggered communication via FlexRay.

- Lack of fail-operational behavior: The basic functionality of a car is still realized with mechanical systems, which are supported by actuators and electronic extensions. In the case of failure, subsystems are stopped in a safe state. This behavior is not desired for future applications like drive-by-wire systems or autonomous driving assistants.

- Increasing demand for interconnection: Predictive active safety functions among all use data-fusion approaches to get a complete state of the environment, as well as the car itself. This means an increasing amount of sensors are involved with high bandwidth and quality-of-service demands.

- Complex system verification: Heterogeneous networks and black-box electronic control units make the system verification process a challenging task. The increasing demand for cross-domain functionality pushes the testing costs even higher.

- Limited flexibility: The ICT in today's cars comes in a static configuration with very limited possibilities to add components after-sale. Additional systems like parking sensors usually come with a dedicated humanmachine interface and are not well-integrated into the existing system. 


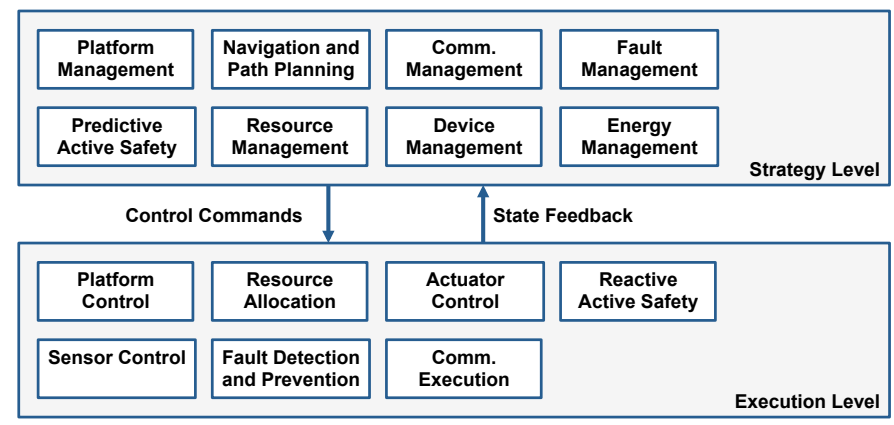

Figure 1. System Architecture.

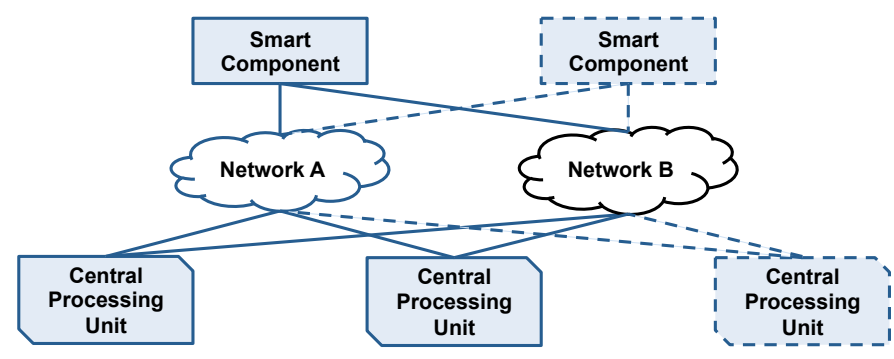

Figure 2. Hardware Architecture.

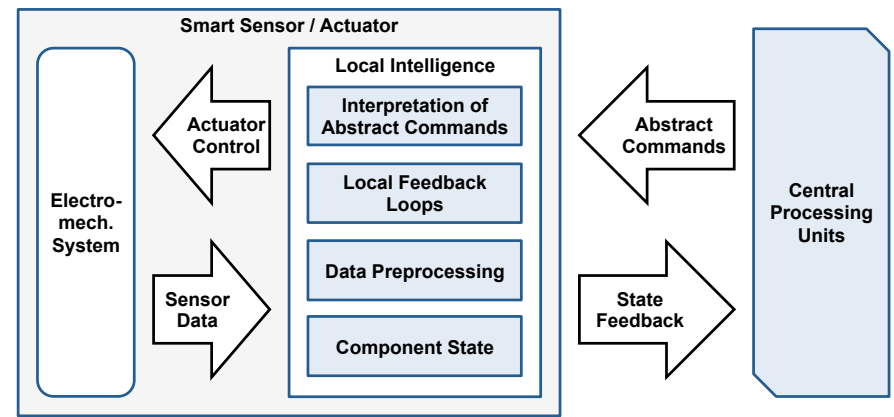

Figure 3. Smart Sensor / Actuator Architecture.

\section{CENTRALIZED ICT ARCHITECTURE}

The centralized architecture combines elements from the system architecture, hardware architecture and software architecture, which together form our concept of a centralized ICT.

\section{A. System Architecture}

The system is designed in a data-driven top-down approach, which focuses on the data flows and processing of the information, instead of the individual components. The aim is to clearly distinguish responsibilities during the development and to minimize the amount of interchanged data. The design principle is separated into two basic levels, see also Fig. 1:

\section{1) Strategy Level}

The aim of the strategy level is to control the execution level and to process the global system state. Knowledge about the environment of the car, the destination and the actual driver's wish are perceived, stored and processed.

\section{2) Execution Level}

The execution level consists of hardware and software components that react on the commands of the strategy level and give feedback of the local state. Components of the execution level should autonomously execute abstract commands sent from the strategy level.

\section{B. Hardware Architecture}

The hardware architecture is divided into central processing units and smart sensors and actuators. Both types of components are interconnected via a deterministic, homogenous and optionally redundant network.

\section{1) Central Processing Units}

The centralized processing units are the deployment point for the high-level functions of the system. Several processing units might be installed in parallel, in order to guarantee safety requirements by redundancy. With standardized interfaces and a set of basic services, the requirements of the software components are matched. Design goals include a flexible system that allows altering, updating and extending the functionality of a vehicle.

\section{2) Smart Sensors and Actuators}

The smart sensors and actuators implement intelligence locally. The data is pre-processed to reduce the busload and a strategy for the execution of abstract commands sent from the central units is implemented, see Fig. 3. Local feedback-loops guarantee fast response times.

\section{Software Architecture}

The software architecture depends on a runtime system which is the backbone of the system that guarantees functional and non-functional requirements. The runtime system is designed to speed up the development and integration process of new functions. The following concepts distinguish our concept from existing systems like AUTOSAR [7]:

\section{1) Data-centric paradigm}

In a data-centric system, each component describes the communication properties it supports and it requires. Together with a naming and data type agreement, the signals are matched by the runtime system or a modeling tool, leading to a virtually decoupled system. With the additional description of non-functional requirements, the runtime system can fulfill quality-of-service requirements by an automatic distribution and data path planning for the requesting components.

\section{2) Resource-Awareness}

As the possibility for on-line updating and reconfiguration is desired, the system has to have knowledge about the required resources of the already running software components like processing time, communication demand and memory amount.

\section{3) Integrated Services}

Base services support the developer and system integrator by offering frequently needed functionality. In the automotive area, this includes generic data-fusion, safety and security concepts. 


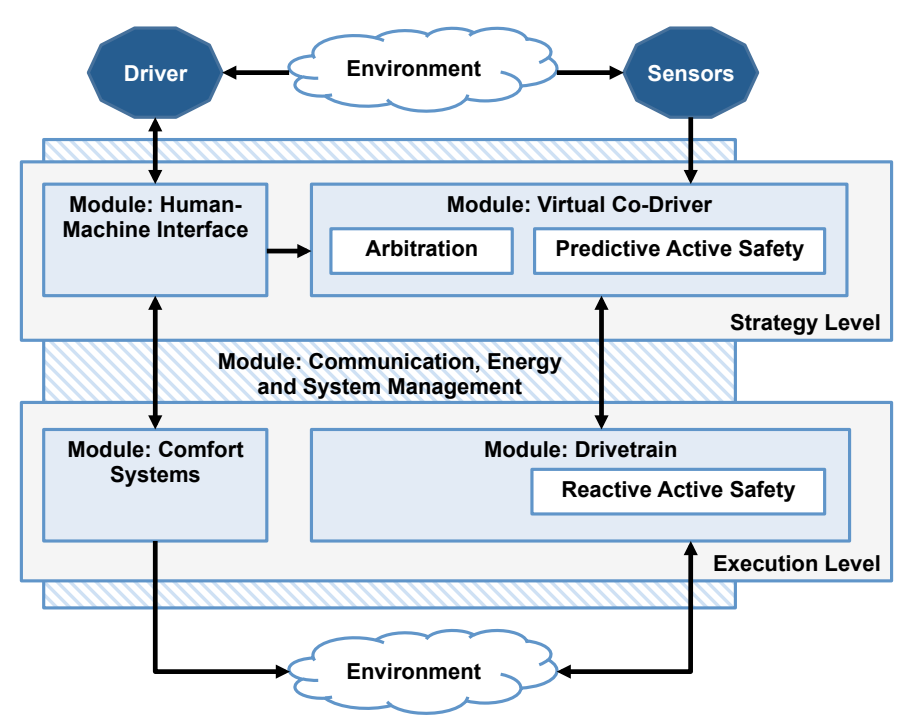

Figure 4. 5-module Concept.

\section{Migration StRATEgIES}

A centralized architecture is a disruptive change to the existing system. Thus, the barrier for such a radical change is very high for existing car manufacturers. The proposed concepts can be used for a stepwise migration to a centralized system:

\section{A. Gateway approach}

For the migration, the architecture can be integrated in a car as an additional, separated system that is connected to the classical architecture via the central gateway. During the evolution of a car, more and more components can be integrated into the centralized approach.

\section{B. 5-module concept}

The 5-module concept was introduced by Prof. Spiegelberg and describes an aggregation of a vehicle's functions into specific modules, see Fig. 4. The aggregation was chosen to match the idea of a data-driven development. Each module represents a software, hardware and business unit. The modules are as follows:

- Strategy level: The strategy level consists of the modules human-machine-interface and virtual co-pilot. An arbitration unit can intervene in the case of danger and override the driver's wish.

- Execution level: The execution level consists of the modules drivetrain and comfort systems. The drivetrain module abstracts all the aggregates involved in vehicle motion and traction.

- Meta level: The meta level consists of one module which is used for the physical interconnection and offers services for the external communication, energy management and system management.

The main difference to the previously described approach is that the individual modules of this concept are heterogeneous and communicate via different physical busses and protocols with the attached sensors and actuators. Although re-branded, this will keep the classic automotive development domains intact. This concept can be migrated to a centralized architecture by the unification of the used busses and the stepwise introduction of the runtime system on the individual modules.

Compared to the established automotive domains in AUTOSAR, the 5-module concept reduces the interconnection demand between the domains. The 5-module concept was also introduced with the efficient usage of business units in mind. If the need for communication is reduced in the physical world, the communication demand between the business units is reduced as well leading to a more robust development process.

\section{Electric mobility}

Electric mobility is a chance for new system architectures as a changed drivetrain concept demands a radical change in the ICT system architecture as well. Small, agile and innovative companies are trying to enter the market of electric mobility that are open to changes in the conservative structures. These companies might be incubators for a change in the system architecture that is later adopted by the established vendors.

\section{ECONOMIC IMPACT}

As a disruptive technology, the initial application of a centralized ICT architecture is cost-intensive but will pay off on the long run:

\section{A. Lowered barrier for market entrance}

With a runtime system that fulfills non-functional requirements, the entrance barrier to develop actuators and sensors for the automotive industry is lowered. This opens the market for small and medium scale companies and causes a higher competition eventually leading to a more customizable car and high quality software components.

\section{B. Resource saving}

The cabling harness is significantly reduced as a homogenous network system avoids the necessarily for several parallel busses deployed inside a car. The centralization of the functions optimizes the energy requirements, since the amount of data transfer on the external buses is reduced.

\section{Faster development cycles}

An architecture that supports updating of components will lead to faster development cycles. A car will always be up-todate and can interconnect to different end-user devices by exchanging the on-board firmware.

\section{Personalization}

As the functions are mainly implemented in software components, the configuration is highly flexible and can be adapted to the user's needs. The configuration can even be transferred between different vehicles offering new market concepts e.g. in the scope of car sharing. 


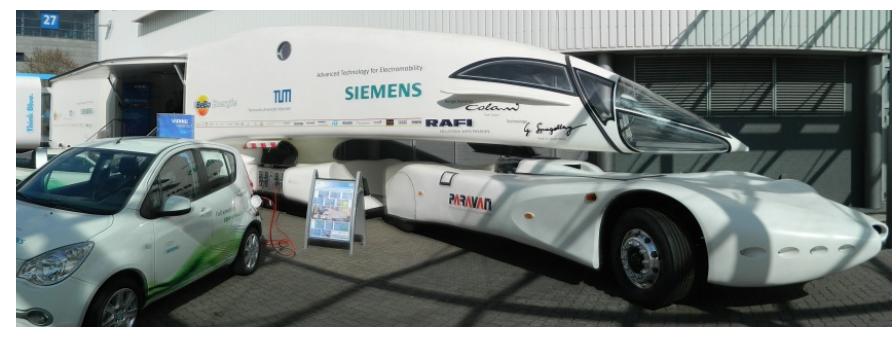

Figure 5. The InnoTruck.

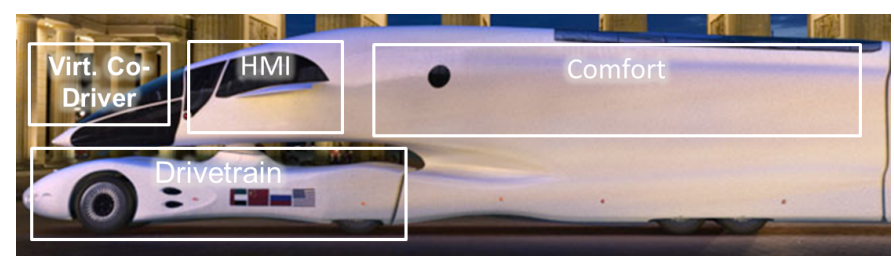

Figure 6. Mapping of Modules to Real Vehicle.

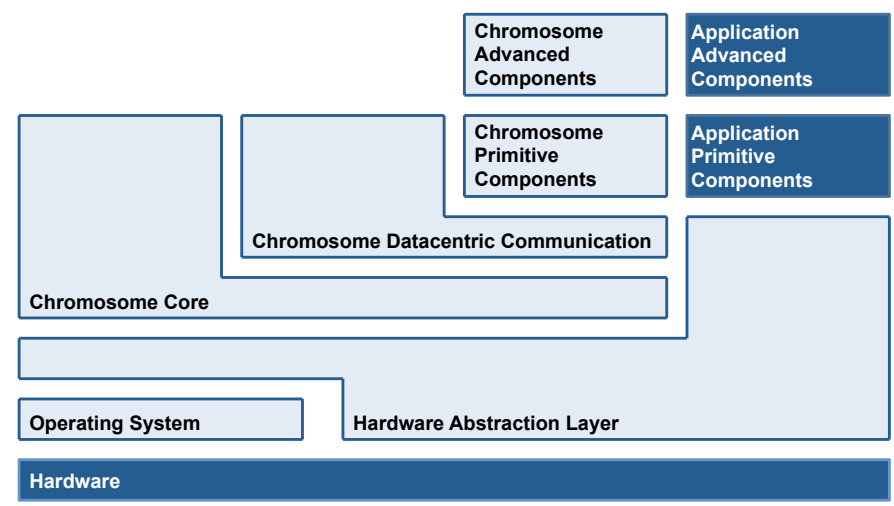

Figure 7. Chromosome Software Architecture.

\section{DEMONSTRATORS}

Several demonstrators are used to proof and evaluate different aspects of the centralized ICT architecture:

\section{A. InnoTruck}

The InnoTruck [3] was developed in the project "Diesel Reloaded" hosted at the International Graduate School for Science and Engineering, see Fig. 5. It is a proof-of-concept and communication platform for the migration strategy of the 5-module architecture. It is a full-scale truck and features a drive-by-wire system and a hybrid drivetrain configuration. Its design allows a direct physical mapping of the theoretical architecture to real-life vehicle modules, see Fig 6 . The management of external and internal energy sources and sinks further tightens the requirements for the ICT architecture.

\section{B. eCar}

The drivable eCar [6] was developed as a platform for future ICT architectures. Its setup features four highly integrated eCorner wheel modules that can be controlled in direction, acceleration and deceleration individually. The eCar implements the introduced hardware architecture with a centralized processing unit and smart sensor and actuators connected to it.

\section{Chromosome}

Chromosome is a runtime system under development, which combines the guarantee of functional and non-functional requirements [4][5]. It is portable to different platforms, uses a data-centric description of the communication requirements, offers a modeling tool to describe a system and can be adapted to different domains. An abstract of the architecture is given with Fig. 7. Key features include:

- Resource-Awareness

- Real-time capabilities

- Support of reconfiguration

- Implicit functions for safety and data-fusion

Chromosome will be used as a runtime system for the demonstrators eCar and InnoTruck in the future.

\section{CONCLUSION}

In this paper, we have extended and refined the previously introduced centralized ICT architecture for future vehicle generations. Problems of existing vehicle architectures were highlighted and a migration path shown to integrate the centralized approach step-by-step. The economic impacts of the new approach were analyzed and demonstrators for proofof-concept and evaluation introduced. Future work will focus on a concrete implementation of the runtime system and an evaluation with a completed vehicle setup.

\section{REFERENCES}

[1] C. Buckl, A. Camek, G. Kainz, C. Simon, L. Mercep, H. Stähle, A. Knoll, "The software car: Building ICT architectures for future electric vehicles," Electric Vehicle Conference (IEVC), 2012 IEEE International, pp.1,8, 4-8 March 2012.

[2] R. N. Charette, "This car runs on code," IEEE Spectrum, vol. Green Tech, Advanced Cars, Feb 2009, available online, http: //spectrum.ieee.org/green- tech/advanced- cars/this- car- runs- oncode $/ 0$.

[3] L. Mercep, C. Buitkamp, H. Stähle, G. Spiegelberg, A. Knoll and M. Lienkamp, "The innotruck case study on a holistic approach to electric mobility," 5th International Conference on Sustainable Automotive Technologies, September 2013, Ingolstadt, Germany, in press.

[4] K. Huang; G. Chen; N. Keddis, M. Geisinger, C. Buckl, "Demo abstract: An inverted pendulum demonstrator for timed model-based design of embedded systems," IEEE/ACM Third International Conference on Cyber-Physical Systems (ICCPS), April 2012 , pp.224,224.

[5] Fortiss GmbH, "CHROMOSOME", 2013, online ressource, http://chromosome.fortiss.org/.

[6] M. Eder and A. Knoll. "Design of an experimental platform for an x-bywire car with four-wheel steering," Proceedings of the 6th IEEE Conference on Automation Science and Engineering, August 2010, pages 656-661.

[7] The AUTOSAR development partnership, "automotive open system architecture (AUTOSAR)", release 4.0, 2013, online available, http://www.autosar.org. 